\title{
G sonstem \\ Tele-Medicine Provides an Effective Tool for the Management of COVID-19 in Children
}

\section{Marco Poeta}

University of Naples Federico II: Universita degli Studi di Napoli Federico II https://orcid.org/0000-00027515-1394

\section{Luca Pierri}

Federico II University Hospital: Azienda Ospedaliera Universitaria Federico II

\section{Eugenia Bruzzese}

Federico II University Hospital: Azienda Ospedaliera Universitaria Federico II

\section{Francesco Nunziata}

Federico II University Hospital: Azienda Ospedaliera Universitaria Federico II

\section{Edoardo Vassallo}

Federico II University Hospital: Azienda Ospedaliera Universitaria Federico II

\section{Andrea Catzola}

Federico II University Hospital: Azienda Ospedaliera Universitaria Federico II

\section{Gian Paolo Ciccarelli}

Federico II University Hospital: Azienda Ospedaliera Universitaria Federico II

Alfredo Guarino ( $\nabla$ alfguari@unina.it )

Federico II University Hospital: Azienda Ospedaliera Universitaria Federico II

\section{Andrea Lo Vecchio}

Federico II University Hospital: Azienda Ospedaliera Universitaria Federico II

\section{Research Article}

Keywords: COVID-19, Children, SARS-CoV-2, Tele-medicine, Tele-health, Model of care

Posted Date: February 15th, 2021

DOl: https://doi.org/10.21203/rs.3.rs-191998/v1

License: (c) (1) This work is licensed under a Creative Commons Attribution 4.0 International License. Read Full License 


\section{Abstract}

Overcrowding, the unavailability of personal protective equipment, and transmission between health care workers are the main causes of the rapid spread of coronavirus disease 2019 (COVID-19) in hospital settings. Because children have less severe symptoms than adults, we used tele-medicine (TM) as the main approach to managing subjects younger than 14 years who contacted their primary care paediatrician (PCP). A TM program for suspected paediatric COVID-19 cases was developed by the COVID-19 paediatric referral centre and PCPs in the Campania Region, Italy. A total of 269 cases of suspected COVID-19 were discussed through tele-consultation between PCPs and paediatric infectious disease specialists. The main reason for the initiation of tele-consultation by PCPs was the need for case management advice ( $n=206,77 \%)$. A total of 203 children were tested for COVID-19, and 139 were positive (73 males, aged 5 years [IQR 1-10]), of whom 103 were managed at home with phone/video monitoring, 17 received a direct medical visit and discharged to home, and 19 were admitted to the COVID-19 unit. The main symptoms were fever (45\%) and cough (25\%). Thirty-five percent were asymptomatic and detected in family clusters. None had severe clinical outcomes.

\section{Conclusion}

TM is a reliable tool to limit the spread of COVID-19 and might reduce the number of unnecessary visits and hospital admissions. Through tele-consultation with an expert, PCPs can identify at-risk children who need testing and manage most cases remotely. However, standardization of the TM approach and criteria for hospital admission are needed.

\section{What Is Known}

- The need for a new model of care has emerged as an important issue with regard to providing effective care and limiting the spread of COVID-19.

- Children have a less severe course of COVID-19 than adults and almost never need an in-person evaluation.

\section{What Is New}

- Tele-visits, tele-consultations and telephone triage are effective tools to limit unnecessary medical visits and COVID-19 testing, allowing the management of children with mild-to-moderate cases as outpatients.

- However, because formal validation has not yet been performed, studies are needed to produce recommendations and guidelines to assist physicians in the implementation of tele-medicine.

\section{Introduction}


Children, especially infants, are susceptible to severe acute respiratory syndrome coronavirus 2 (SARSCoV-2) infection, although the clinical manifestations are generally less severe than those in adults (1). Available data suggest that children may exhibit more upper respiratory tract involvement, and asymptomatic infections are common (2). The clinical features range widely, from fever, cough, and pharyngeal erythema to severe dyspnoea, hypoxemia, and acute respiratory distress syndrome.

Italy was the first European country affected by the pandemic. At the onset of the epidemic, transmission was largely hospital-based, and there was little or no difference in the management of children and adults. In 2020 , more than $10 \%$ of all SARS-CoV-2 infections were detected in subjects younger than 19 years, with twenty-one deaths in this age group (3). The acceleration of the coronavirus disease 19 (COVID-19) pandemic poses a major challenge for all health care systems, with demand exceeding their maximum capacity for a protracted duration in the absence of sufficient resources. These resources include health care workers, who are at high risk for infection and account for 10 to $20 \%$ of COVID-19 patients and COVID-19-related deaths (4).

New models of care are needed to effectively counteract COVID-19, reduce overcrowding in hospitals, and reduce the spread of the infection to health care personnel (5). Tele-medicine (TM) could be an appropriate tool for the management of communicable diseases owing to its efficacy at reducing personto-person transmission (6). TM improves access to health care services, especially in sparsely populated and less-developed regions, by facilitating contact between patients and providers. TM has been widely tested over the past 20 years and provides an easy-to-establish and accessible alternative to direct health care delivery (7). In particular, in the setting of non-urgent/routine care, remote assessment provides an opportunity for social distancing and triaging between routine or emergency access to congested hospitals, thereby reducing the risk of infection (8). Phone consultations can be used by health care workers to replace, or supplement, standard medical visits for several patient needs. Physicians can interact with patients over the telephone or via other electronic devices, collecting the relevant patient history and key information on health concerns, evaluating symptoms to some extent, and offering advice (9). Images and videos can supplement the conversation to provide further clinically useful information.

In addition to its use for chronic conditions, TM has been useful during previous outbreaks, including coronavirus outbreaks such as those of SARS and MERS (Middle East respiratory syndrome) or public health emergencies related to the Ebola and Zika viruses (10). In 2019, in the United Kingdom, the high burden imposed by the seasonal influenza outbreak led to the implementation of innovative TM-based solutions as a first-line approach to reduce patient visits to the emergency department (11). The application of TM during outbreaks facilitates tele-consultation, which can be indispensable in primary and secondary care settings when local medical resources need support from referral centres. Through tele-consultation, clinicians may benefit from expert advice as well as peer-to-peer approaches.

A remote health surveillance model based on telephone triage (TT) has been successful at controlling the spread of seasonal influenza (12), suggesting that some COVID-19 patients can be managed at a 
distance via remote consultation. Individuals who fear they may be infected with SARS-CoV-2 may benefit from remote consultations, reducing unnecessary direct medical intervention. Technological improvements, access to high-speed internet, and the widespread availability of smartphones have already enabled the rapid and easy implementation of tele-consultations for adult patients during the COVID-19 pandemic (13).

During the first phase of the epidemic, Italy did not include TM in essential care; in contrast, TM consultation systems were rapidly established in China (14), France (15), the United States (16) and Brazil (17) to prevent COVID-19 from spreading and were shown to be effective with regard to remote patient monitoring, with low rates of direct medical visits and hospital admissions.

The relatively mild clinical course in most children with COVID-19 offers a potentially ideal opportunity for the socially distanced management of children through the implementation of $\mathrm{TM}$, which may contribute to containment of the spread of the epidemic. The COVID-19 peak occurred later in the region of Campania than in northern Italy. A paediatric hub centre located in the Paediatric Infectious Disease Unit of the University Hospital and linked with the paediatric primary care system operated in cooperation with primary care paediatricians (PCPs) through TM. In Italy, the preventive and primary care of all children are managed by PCPs (i.e., family paediatricians), who have full knowledge of the family setting, including the clinical history, health status, and social and living conditions of the children under their care (18). Each PCP is in charge of 1000 children.

The aim of this study was to investigate the efficacy and feasibility of TM, in the form of teleconsultation, for the management of suspected and confirmed paediatric COVID-19 cases. The project was implemented in cooperation with PCPs and experts in paediatric infectious diseases from the paediatric COVID-19 centre of the University Federico II of Naples, Italy.

\section{Methods}

\section{Study design and participants}

A specific program was designed and assessed during the COVID-19 pandemic (March to December 2020). The regional referral centre for COVID-19 in children coordinated the management of children in the Campania region, which has five million residents and approximately 500,000 children. A telephone number was available to PCPs at all times that they could call for advice and to discuss clinical indications, as well as for triage by the hospital and the admission of children, if necessary. PCPs received a weekly letter from a referral centre containing updated reports on paediatric COVID-19 and the indications for testing and/or hospital admission. Upon request by the family of children with suspected cases of COVID-19, PCPs adapted their tele-visit program to include the caregivers of the children. For support when making clinical decisions, PCPs used tele-consultation, a specific "clinician-to-clinician" TM approach, which allowed them to confer with paediatric specialists in infectious diseases at the referral centre. 
Children aged 0-14 years were enrolled through tele-consultation between PCPs and experts at the referral centre, who performed a TT to define the need for screening and identify the most suitable strategy for managing the case (home isolation, in-person visit, swab and/or other investigations or hospital admission). Experts at the COVID-19 unit provided updated information about indications to the PCPs and discussed all cases with regard to the signs and symptoms, exposure history, and risk of COVID-19 transmission within the household, particularly to members at an increased risk of severe disease (i.e., subjects aged $\geq 65$ years with chronic conditions). In addition, they critically evaluated the efficacy of home isolation measures. Children managed at home were monitored daily by PCPs through tele-visits. Follow-up contact was established between the referral centre and PCPs if major clinical changes, concerning signs, or spread among contacts occurred.

\section{Data collection}

Data were collected through a semi-structured telephone interview with PCPs. We recorded the following patient information in an anonymized dataset: sex, age, date of symptom onset, contact history, members of the household, and ongoing therapies. In addition, we collected data during phone consultations between PCPs and the experts at the referral centre, particularly regarding the mean duration of symptoms and main reasons for the phone call (case management, reassurance, indications for diagnostic tests, home isolation/quarantine rules, and need for hospital admission). Furthermore, to evaluate the feasibility and efficacy of the TM approach for selecting the appropriate management strategies for patients who potentially needed hospitalization, admitted children referred by PCPs through tele-consultation were compared with a sample of 69 children admitted after a direct medical visit in emergency departments (EDs) who were not engaged in the TM-based protocol. The appropriateness of hospital admissions was evaluated at discharge based on the occurrence during the hospital stay of need for clinical/laboratory monitoring, medical procedures and parenteral therapies and disease progression.

\section{Statistical analysis}

Analyses were performed with IBM SPSS Statistics for Windows, Version 26.0 (Armonk, NY: IBM Corp). Data are presented as the medians (IQRs), and categorical variables are presented as the frequencies (\%). Fisher's exact tests and Mann-Whitney tests were used to compare the frequencies of events and nonnormally distributed variables.

\section{Result}

The specific TM program for the management of children with suspected cases of COVID-19 is schematically depicted in Figure 1. A total of 270 telephone contacts with 162 PCPs were recorded. At the beginning of the epidemic, knowledge of the disease was limited. The risk of the spread of infection was not a major consideration when designing management plans, and generally, children were perceived by both the families and PCPs as being at high clinical risk rather than sources of COVID-19 transmission. However, after contact with experts and as the pandemic progression, the transmission of the infection 
became a major determinant of medical decisions. The primary reason for telephone contact was the need for case management advice $(n=206,77 \%)$, followed by diagnostic test indications $(n=38,14 \%)$. Sixty-six children without suggestive clinical features and/or exposure history to COVID-19 did not match the indications for COVID-19 testing (25\%). COVID-19 testing was recommended and performed for 203 children, of whom 139 were positive (51\%) and 64 were negative (24\%) (Table 1).

The epidemiological and clinical features of the children with COVID-19 are reported in table 2 and table 3, respectively. COVID-19 patients are presented as the total $(n=139)$, those managed with TM $(n=103$, $74 \%)$, those who received in-person triage $(n=17,12 \%)$ and those admitted to the hospital $(n=19,14 \%)$. As expected, there were more symptomatic children in the cohort of hospitalized patients $(p=0.006)$, and in most cases, the clinical presentation was similar to a flu-like syndrome.

As assessed by TM, the median number of household members was four (IQR 3-5). Eighty-two (59\%) patients had at least one member of the household with symptoms (fever, cough), and 110 (79\%) had one or more members of the household who had tested positive for COVID-19. The main clinical features in the children with COVID-19 were fever $(n=63,45 \%)$ and cough $(n=35,25 \%)$. Forty-nine were asymptomatic $(49 \%)$ and were tested because there were clusters of cases in their families.

Direct medical visits by PCPs were not necessary for any of the patients managed at home, who did well without major complications, as indicated by the absence of the development of further problems or additional follow-up requested. However, in one case, after multiple telephone contacts with the PCP, a 2month-old infant who initially managed through TM at home because of the absence of symptoms, was admitted to the hospital due to the development of fever and mild respiratory distress. This was actually the first child admitted to the hospital for COVID-19, when the clinical experience and knowledge of the natural history of the diseases were virtually non-existent. Nine asymptomatic patients were triaged in the hospital due to the difficulty of performing swab tests at home. Age $<1$ year, fever and respiratory distress were more common in patients admitted to the hospital than in children managed at home $(p<0.05)$. The median age was younger in children admitted to the hospital, although the difference was not statistically significant. Children not engaged in the TM program were referred for hospital isolation at the regional COVID-19 unit after accessing the EDs of regional hospitals $(n=69)$. As shown in Table 4, there were no significant differences (age, presence of symptoms and main reason for hospitalization) between children hospitalized after being seen in EDs and those referred by PCPs through tele-consultation. A substantial number of admissions were eventually deemed unnecessary, without a statistically significant difference between the two groups ( $38 \%$ and $27 \%$, respectively, $p>0.05$ ). These patients, even though they were admitted to the hospital, did not have severe cases and did not require parenteral therapies, specific hospital-based procedures or isolation.

\section{Discussion}

TM, including TT, is emerging as an essential component of the health care system that is applicable for the management of many diseases and has been proposed as a key clinical approach during pandemics 
(10). The need for new TM models of care suitable for paediatric patients was supported by the increased number of weekly tele-visits to "My Health at Vanderbilt", a TM project active in Tennessee (USA), at the start of the COVID-19 epidemic (19). In addition, a TM approach has been successfully applied to provide uninterrupted care for children with asthma during pandemics (20).

Our data confirm a less severe course of COVID-19 in children than in adults (1), making it feasible to manage most paediatric patients at home through phone or video calls. This approach was highly effective because it involved PCPs who have full knowledge of the clinical and psychosocial conditions of children of whom they are in charge in the national health care system and their families. PCPs could also effectively collect data on exposure, pre-existing conditions, ongoing therapies, the presence of features possibly indicating a condition other than COVID-19, and red flags indicating the need for prompt direct assessment. The long-term knowledge of the child and the close relationship with the family help PCPs foster trust on the part of the families that they are correctly interpreting the information obtained at distance and, in turn, increase the trust of caregivers in the effectiveness of home management according to the indications remotely provided by their physician. In our experience, direct assessment was needed only in rare circumstances. Despite the lack of full and direct physical examinations, PCPs could easily obtain information on the paediatric patient's body temperature and oxygen saturation through the tools available at the patient's home. General appearance, respiratory distress signs (nasal flaring, sternal and intercostal retractions) and respiratory rates were obtained through visual evaluation and the use of instant messaging platforms, as previously discussed (21). Telephone calls and the implementation of technologies that facilitate remote management limit patient access to PCP offices and EDs, resulting in many beneficial effects, the most significant being a reduction in the spread of the virus, in which paediatric patients could play a key role. Although children are less likely to develop symptoms and are tested at relatively lower rates than adults, they may contribute to the spread of the infection, particularly to vulnerable populations (22).

The sharing of expertise through tele-consultation appears effective in complex medical situations. An example can be found in the ECHO (Extension for Community Health care Outcomes) Project, coordinated by the University of New Mexico Health Sciences Center, which links interdisciplinary specialist teams with primary care clinicians through a virtual platform. The ECHO Project has been effectively used for the management of numerous conditions, ranging from hepatitis $C$ to behavioural health conditions (23). In our experience, tele-consultation between PCPs and specialists at the referral centre has emerged as an effective tool for the management of acute infectious diseases such as COVID19 , reducing over-testing and over-treatment. In fact, a number of children were referred to the centre because of the fear of contagion and were excluded from testing after tele-consultation. No cases of COVID-19 were eventually diagnosed among them, indirectly suggesting the reliability of tele-consultation for the identification of suspected cases.

Considering the less severe course in the paediatric patient population (24), children only rarely need direct clinical evaluation and can be monitored through tele-visits, limiting the exposure of health care professionals. In our model, tele-consultation and, consequently, the recommendation for home isolation 
reduced unnecessary contact, thereby decreasing the risk of transmission from infected children in health care settings and minimizing the consumption of personal protective equipment (4). Furthermore, TM reduces intrafamilial transmission, particularly transmission of the virus to vulnerable household members (22).

To date, only nineteen out of 139 children with COVID-19 referred by PCPs have been admitted to our paediatric COVID-19 unit, and in many children, hospitalization was eventually determined to have not been needed. Cases remotely managed by PCPs through TM, with daily phone calls and the opportunity to consult paediatric infectious disease experts through subsequent tele-consultations, had good clinical outcomes, and hospital admission was necessary in only one case during follow-up due to the clinical deterioration of the patient.

The younger age of admitted children suggests that the need for hospitalization was frequently due to fear and worry due to limited COVID-19 knowledge rather than severe clinical manifestations related to the unpredictable course of the disease. Indeed, all referred cases had a favourable course regardless of the management setting. Certainly, age below 3 months in the presence of warning signs (i.e., dyspnoea, dehydration, fever, comorbidities) should still be considered an indication for admission to rule out coinfections and a more severe course of COVID-19. However, we need evidence-based criteria for admission stratified by age group.

An important advantage of our TM model is the reduction in unnecessary clinical visits and hospital admissions. In fact, most admitted children were referred to the centre from EDs, and they did not undergo TT with PCPs. This led to unnecessary hospitalization mainly due to the need to limit the spread of COVID-19 rather than because they required clinical management. Nevertheless, TM is not a perfect tool, considering the relatively high burden of unnecessary admissions among PCP-referred children through tele-consultation or among patients admitted from EDs. Although the limited sample size prevents firm conclusions from being drawn, our results suggest that remote management by PCPs leads to decisions similar to those made with direct management in EDs in this specific population. Conversely, through the implementation of TM in primary care, patients who needed hospitalization were promptly screened at home for COVID-19, avoiding unnecessary contact with patients at the hospital who did not have COVID-19 and healthcare workers in EDs. Finally, hospital management was performed in specific paediatric settings by highly trained and equipped medical staff. None of the 20 healthcare providers working in the COVID-19 paediatric unit were infected, unlike in adult patient units, where substantial infection rates were reported among healthcare personnel (25).

Tele-visits and video contacts allow sufficiently accurate assessments in most cases, reducing exposure to a range of infectious diseases other than COVID-19 (26); however, clinicians should always use their judgement to critically evaluate the reliability of caregivers (27). Of note, caregivers, mainly the parents of the children, demonstrated a collaborative attitude and reacted well to the TM approach. Here, TM was successfully applied to promote social distancing and protect vulnerable children with chronic diseases 
during the COVID-19 outbreak; however, the implications for their health status and the evaluation of quality of care in these settings should be considered in the long term.

\section{Conclusion}

TM for COVID-19 in children may be easily and quickly implemented and is highly effective when adopted in conjunction with a child-specific model of primary care (18). As shown in adults (14-17), our experience confirms that distanced care is a safe alternative to in-person medical care for children with mild-tomoderate symptoms, reducing the spread of infection (22), optimizing the use of financial and human resources (28), preventing psychological distress in caregivers (29), avoiding hospital overcrowding, reducing the consumption of personal protective equipment (30), and maintaining the well-being of healthcare providers (31). Once sufficient data and standard pathways are available, TM could be included in protocols for managing children exposed to or infected with SARS-CoV-2.

Studies to validate a systematic approach to uniformly applying tele-visits to the management of paediatric cases of COVID-19 need to take into account socio-economic family inequities with regard to accessing healthcare services (32).

\section{Abbreviations}

COVID-19: coronavirus disease 2019

ED: Emergency Department

MERS: Middle East respiratory syndrome

PCP: primary care paediatrician

SARS-CoV-2: severe acute respiratory syndrome coronavirus 2

TM: tele-medicine

TT: telephone triage

\section{Declarations}

Funding: This work was supported by a donation from Yamamay®.

Conflicts of interest/Competing interests: The authors declare that the research was conducted in the absence of any commercial or financial relationships that could be construed as a potential conflict of interest.

Ethics approval: Patients were included in a clinical, immunological and genetic study reviewed and approved by the ethics committee of the University of Naples Federico II (April 22, 2020/No. 158/20). The 
study was performed in accordance with the Declaration of Helsinki for Human Research.

Consent to participate: Written informed consent was obtained from the parents of the children admitted to the hospital. Verbal informed consent was obtained from the parents of the children managed via telemedicine.

Consent for publication: Written informed consent was obtained from the parents of the children admitted to the hospital. Verbal informed consent was obtained from the parents of the children managed via tele-medicine.

Availability of data and material: The datasets generated for this study are available on request to the corresponding author.

Code availability: N/A

Authors' contributions: MP and ALV were involved in the study design and contributed equally to the project. EB, FN, GPC, and AC collaborated to implement the different phases of the study and to collect the data. LP and EV performed the statistical analysis and prepared the tables and figures. AG was the guarantor of the manuscript and supervised the drafting of the manuscript. All authors have read and approved the final version of the manuscript.

Acknowledgements: We would like to thank all primary care paediatricians who contributed to the implementation of the tele-medicine project. We also thank all our colleagues involved in the area rescue teams for their contributions.

\section{References}

1. Dong Y, Mo X, Hu Y, et al (2020) Epidemiology of COVID-19 Among Children in China. Pediatrics. pii: e20200702. doi: 10.1542/peds.2020-0702.

2. Lu X, Zhang L, Du H, et al (2020) SARS-CoV-2 Infection in Children. N Engl J Med. 382:1663-1665. doi: 10.1056/NEJMc2005073.

3. Epidemiology for public health (2020) COVID-19 integrated surveillance: key national data. https://www.epicentro.iss.it/en/coronavirus/sars-cov-2-integrated-surveillance-data. Accessed 14 January 2021

4. The Lancet (2020) COVID-19: protecting health-care workers. Lancet 395:922. doi: 10.1016/S01406736(20)30644-9.

5. Greenhalgh T, Koh GCH, Car J (2020) Covid-19: a remote assessment in primary care. BMJ 368:m1182. doi: 10.1136/bmj.m1182.

6. Spooner SA, Gotlieb EM (2004) Telemedicine: pediatric applications. Pediatrics 113:e639-e643. doi: 10.1542/peds.2015-1517. 
7. Zanaboni P, Wootton R (2016) Adoption of routine telemedicine in Norwegian hospitals: progress over 5 years. BMC Health Serv Res 16:496. doi: 10.1186/s12913-016-1743-5.

8. Smith AC, Thomas E, Snoswell CL, et al (2020) Telehealth for global emergencies: Implications for coronavirus disease 2019 (COVID-19). J Telemed Telecare 26:309-313. doi: $10.1177 / 1357633 \times 20916567$.

9. Lucero-Obusan C, Winston CA, Schirmer PL, Oda G, Holodniy M (2017) Enhanced Influenza Surveillance Using Telephone Triage and Electronic Syndromic Surveillance in the Department of Veterans Affairs, 2011-2015. Public Health Rep 132:16S-22S. doi: 10.1177/0033354917709779

10. Ohannessian R, Duong TA, Odone A (2020) Global Telemedicine Implementation and Integration Within Health Systems to Fight the COVID-19 Pandemic: A Call to Action. JMIR Public Health Surveill 6:e18810. doi: 10.2196/18810

11. Romm S. (2019) Telemedicine Emerging as a First Line of Defense during Flu Season. https://www.physicianspractice.com/article/telemedicine-emerging-first-line-defense-during-fluseason. Accessed 14 January 2021

12. Nagykaldi Z, Calmbach W, DeAlleaume L, Temte J, Mold J, Ryan JG (2010) Facilitating patient selfmanagement through telephony and web technologies in seasonal influenza. Informatics in Primary Care. 18:9-16. doi: 10.14236/jhi.v18i1.749.

13. Khairat S, Meng C, Xu Y, Edson B, Gianforcaro R (2020) Interpreting COVID-19 and Virtual Care Trends: Cohort Study. JMIR Public Health Surveill 6:e18811. doi:10.2196/18811

14. Ren X, Zhai Y, Song X, Wang Z, Dou D, Li Y (2020) The Application of Mobile Telehealth System to Facilitate Patient Information Presentation and Case Discussion. Telemed J E Health 26:725-733. doi: $10.1089 / \mathrm{tmj} .2020 .0084$.

15. gouv.fr. (2020) Décret n²020-227 du 9 mars 2020 adaptant les conditions du bénéfice des prestations en espèces d'assurance maladie et de prise en charge des actes de télémédecine pour les personnes exposées au Covid-19. https://www.legifrance.gouv.fr/affichTexte.do? cidTexte=JORFTEXT000041704122\&categorieLien=id Accessed 14 January 2021

16. Mann DM, Chen J, Chunara R, Testa PA, Nov O (2020) COVID-19 transforms health care through telemedicine: Evidence from the field. J Am Med Inform Assoc 27:1132-1135. doi: 10.1093/jamia/ocaa072.

17. Nascimento BR, Brant LC, Castro ACT, et al (2020) Impact of a large-scale telemedicine network on emergency visits and hospital admissions during the coronavirus disease 2019 pandemic in Brazil: Data from the UNIMED-BH system. J Telemed Telecare 25:1357633X20969529. doi: $10.1177 / 1357633 \times 20969529$.

18. Corsello G, Ferrara P, Chiamenti G, Nigri L, Campanozzi A, Pettoello-Mantovani M (2016) The Child Health Care System in Italy. J Pediatr 177S:S116-S126. doi: 10.1016/j.jpeds.2016.04.048.

19. Patel PD, Cobb J, Wright D, et al (2020) Rapid Development of Telehealth Capabilities within Pediatric Patient Portal Infrastructure for COVID-19 Care: Barriers, Solutions, Results. J Am Med Inform Assoc pii:ocaa065. doi: 10.1093/jamia/ocaa065. 
20. Wall-Haas CL (2020) Connect, Engage: Televists for Children With Asthma During COVID-19. J Nurse Pract doi: 10.1016/j.nurpra.2020.08.027.

21. Greenhalgh T, Wherton J, Shaw S, Morrison C (2020) Video consultations for COVID-19. BMJ 368:m998. doi: 10.1136/bmj.m998.

22. Kelvin AA, Halperin S (2020) COVID-19 in children: the link in the transmission chain. Lancet Infect Dis. pii:S1473-3099(20)30236-X. doi: 10.1016/S1473-3099(20)30236-X.

23. Arora S, Kalishman S, Dion D, et al (2011) Partnering urban academic medical centers and rural primary care clinicians to provide complex chronic disease care. Health Aff (Millwood). 30:11761184. doi: $10.1377 /$ hlthaff.2011.0278

24. Glauser W (2020) Proposed protocol to keep COVID-19 out of hospitals. CMAJ 192:E264-E265. doi: 10.1503/cmaj.1095852.

25. Remuzzi A, Remuzzi G (2020) COVID-19 and Italy: what next? Lancet 395:1225-1228.

26. Rockwell KL, Gilroy AS (2020) Incorporating telemedicine as part of COVID-19 outbreak response systems. Am J Manag Care 26:147-148. doi: 10.37765/ajmc.2020.42784.

27. Portnoy J, Waller M, Elliott T (2020) Telemedicine in the Era of COVID-19. J Allergy Clin Immunol Pract 8:1489-1491. doi: 10.1016/j.jaip.2020.03.008.

28. Mannelli C (2020) Whose life to save? Scarce resources allocation in the COVID-19 outbreak. J Med Ethics pii:medethics-2020-106227. doi: 10.1136/medethics-2020-106227.

29. Yuan R, Xu QH, Xia CC, et al (2020) Psychological status of parents of hospitalized children during the COVID-19 epidemic in China. Psychiatry Res 288:112953. doi: 10.1016/j.psychres.2020.112953.

30. Chirico F, Nucera G, Magnavita N (2020) COVID-19: Protecting Healthcare Workers is a priority. Infect Control Hosp Epidemiol 41:1117 doi: 10.1017/ice.2020.148.

31. Moazzami B, Razavi-Khorasani N, Dooghaie Moghadam A, Farokhi E, Rezaei N (2020) COVID-19 and telemedicine: Immediate action required for maintaining healthcare providers well-being. J Clin Virol 126:104345. doi: 10.1016/j.jcv.2020.104345

32. Eberly LA, Kallan MJ, Julien HM, et al (2020) Patient Characteristics Associated With Telemedicine Access for Primary and Specialty Ambulatory Care During the COVID-19 Pandemic. JAMA Netw Open 3:e2031640. doi: 10.1001/jamanetworkopen.2020.31640.

\section{Tables}

\section{Table 1. Telephone contacts with the referral center}




\begin{tabular}{|lll|}
\hline Primary Care Pediatricians, $\mathrm{n}$. & 162 & \\
\hline Median contacts/month, n. (IQR) & 26 & $(15-67)$ \\
\hline First telephone contact, $\mathrm{n}$. & 269 & \\
\hline Second telephone contact, $\mathrm{n}$. & 171 & \\
\hline Median duration of phone calls, minutes (IQR) & 10 & $(8-15)$ \\
\hline Type of request, $\mathrm{n}$. (\%) & & \\
Case management & 206 & $(77)$ \\
Reassurance & 13 & $(5)$ \\
Indications to diagnostic test & 38 & $(14)$ \\
Clarifications about quarantine rules & 12 & $(4)$ \\
Covid-19 swab test, $\mathrm{n}$. (\%) & & \\
\hline Positive & 139 & $(51)$ \\
Negative & 64 & $(24)$ \\
\hline Not performed & 66 & $(25)$ \\
\hline
\end{tabular}

Abbreviations. n., number; IQR, interquartile range.

Table 2. Epidemiological features of COVID-19 positive children 


\begin{tabular}{|c|c|c|c|c|c|c|c|c|c|}
\hline \multirow[b]{2}{*}{ Median Age (years) (IQR) } & \multicolumn{2}{|c|}{$\begin{array}{l}\text { Overall } \\
\text { positive } \\
\text { cases } \\
(n=139)\end{array}$} & \multicolumn{2}{|c|}{$\begin{array}{l}\text { Managed in } \\
\text { telemedicine } \\
(n=103)\end{array}$} & \multicolumn{2}{|c|}{$\begin{array}{l}\text { In-person } \\
\text { triaged } \\
(n=17)\end{array}$} & \multicolumn{2}{|c|}{$\begin{array}{l}\text { Hospital } \\
\text { admitted } \\
(n=19)\end{array}$} & \multirow{2}{*}{$\begin{array}{l}p \\
0.435\end{array}$} \\
\hline & 5 & $\begin{array}{l}(1- \\
10)\end{array}$ & 5 & $(1-10)$ & 3 & $\begin{array}{l}(1- \\
10)\end{array}$ & 1 & $\begin{array}{l}(0- \\
6)\end{array}$ & \\
\hline \multicolumn{10}{|l|}{ Age distribution, n. (\%) } \\
\hline$<1$ year & 39 & $(28)$ & 25 & $(24)$ & 4 & $(23)$ & 10 & (53) & 0.050 \\
\hline $1-5$ years & 41 & $(29)$ & 30 & $(30)$ & 7 & $(41)$ & 4 & (21) & 0.439 \\
\hline 6-10 years & 31 & $(22)$ & 27 & $(26)$ & 3 & (18) & 1 & (5) & 0.109 \\
\hline $11-14$ years & 28 & $(21)$ & 21 & $(20)$ & 3 & $(18)$ & 4 & (21) & 1.000 \\
\hline Male, n. (\%) & 73 & (53) & 52 & $(50)$ & 11 & $(65)$ & 10 & (53) & 0.549 \\
\hline \multicolumn{10}{|l|}{ Household and history of contact } \\
\hline $\begin{array}{l}\text { Housemates, median number } \\
\text { (IQR) }\end{array}$ & 4 & $(3-5)$ & 4 & $(3-5)$ & 3 & $\begin{array}{l}(3- \\
5)\end{array}$ & 4 & $\begin{array}{l}(3- \\
5)\end{array}$ & 0.633 \\
\hline $\begin{array}{l}\text { At least one housemate with } \\
\text { symptoms, n. (\%) }\end{array}$ & 82 & (59) & 59 & (57) & 9 & (53) & 14 & (73) & 0.366 \\
\hline $\begin{array}{l}\text { At least one housemate with } \\
\text { confirmed COVID-19, n. (\%) }\end{array}$ & 110 & (79) & 82 & (80) & 15 & (88) & 13 & (68) & 0.350 \\
\hline $\begin{array}{l}\text { Contacts with COVID-19 cases } \\
\text { outside of the household, } n \text {. (\%) }\end{array}$ & 56 & (40) & 44 & (43) & 8 & (47) & 4 & (21) & 0.168 \\
\hline
\end{tabular}

Abbreviations. IQR, interquartile range; $n$., number.

Table 3. Clinical features of COVID-19 positive children and treatment administered 


\begin{tabular}{|c|c|c|c|c|c|c|c|c|c|}
\hline & \multicolumn{2}{|c|}{$\begin{array}{l}\text { Overall positive } \\
\text { cases } \\
(n=139)\end{array}$} & \multicolumn{2}{|c|}{$\begin{array}{l}\text { Managed in } \\
\text { telemedicine } \\
(n=103)\end{array}$} & \multicolumn{2}{|c|}{$\begin{array}{l}\text { In-person } \\
\text { triaged } \\
(n=17)\end{array}$} & \multicolumn{2}{|c|}{$\begin{array}{l}\text { Hospital } \\
\text { admitted } \\
(n=19)\end{array}$} & \multirow[t]{2}{*}{$p$} \\
\hline \multicolumn{9}{|l|}{ Clinical features } & \\
\hline Symptomatic, n. (\%) & 90 & $(65)$ & 63 & (61) & 8 & (47) & 18 & (95) & 0.006 \\
\hline Fever & 63 & $(45)$ & 42 & (41) & 7 & (41) & 15 & (79) & 0.008 \\
\hline Cough & 35 & $(25)$ & 23 & (22) & 4 & (24) & 8 & (42) & 0.186 \\
\hline $\begin{array}{l}\text { Nasal } \\
\text { discharge/rhinorrhea }\end{array}$ & 19 & (14) & 10 & (10) & 4 & (24) & 5 & (26) & 0.045 \\
\hline Myalgia/asthenia & 13 & (9) & 10 & (10) & 1 & (6) & 2 & (11) & 1.000 \\
\hline Gl symptoms & 12 & (9) & 8 & (8) & 2 & (12) & 2 & (11) & 0.679 \\
\hline Sore throat & 10 & (7) & 8 & (8) & 1 & (6) & 1 & (5) & 0.248 \\
\hline Skin lesions & 9 & (6) & 6 & (6) & 2 & (12) & 1 & (5) & 0.637 \\
\hline Respiratory distress & 7 & (5) & 2 & (2) & 2 & (12) & 3 & (16) & 0.019 \\
\hline Dysgeusia/Anosmia & 6 & (4) & 4 & (4) & 1 & (6) & 1 & (5) & 0.490 \\
\hline Conjunctivitis & 2 & $(1)$ & 1 & (1) & 1 & (6) & 0 & (0) & 0.248 \\
\hline Headache & 2 & $(1)$ & 2 & (2) & 0 & (0) & 0 & (0) & 1.000 \\
\hline Asymptomatic, n. (\%) & 49 & (35) & 40 & (39) & 9 & (53) & 1 & (5) & 0.006 \\
\hline \multicolumn{10}{|l|}{ Therapies } \\
\hline Paracetamol & 71 & (51) & 51 & (50) & 7 & (41) & 13 & (68) & 0.217 \\
\hline $\begin{array}{l}\text { Bronchodilator with } \\
\text { spacer }\end{array}$ & 8 & (6) & 5 & (5) & 2 & (12) & 1 & (5) & 0.321 \\
\hline Azithromycin & 22 & $(16)$ & 19 & (18) & 1 & (6) & 2 & (11) & 0.334 \\
\hline Beta-lactams & 15 & (11) & 12 & (11) & 1 & (6) & 2 & (11) & 0.777 \\
\hline Steroids & 6 & (4) & 5 & (5) & 1 & (6) & 0 & $(0)$ & 0.815 \\
\hline
\end{tabular}

Abbreviations. IQR, inter-quartile range; n., number.

Table 4. Comparison of hospital admitted children referred by PCPs and children hospital admitted because of direct EDs access. 


\begin{tabular}{|llll|}
\hline & $\begin{array}{l}\text { Referred by } \\
\text { PCPs } \\
(\mathbf{n = 1 9 )}\end{array}$ & $\begin{array}{l}\text { Admitted after EDs } \\
\text { access } \\
(\mathbf{n}=69)\end{array}$ & $\mathbf{p}$ \\
\hline Median Age (years) (IQR) & $1(0-6)$ & $1(0-4)$ & 0.194 \\
\hline Symptomatic cases, n. (\%) & $18(95)$ & $58(84)$ & 0.449 \\
Asymptomatic cases, n. (\%) & $1(5)$ & $11(16)$ & 0.449 \\
Main reason of hospital admission, n. (\%) & & & 0.038 \\
\hline Clinical features & $14(74)$ & $31(45)$ & 0.130 \\
Pre-existing conditions & $0(0)$ & $8(12)$ & 0.274 \\
Age <3 months & $4(21)$ & $26(38)$ & 1.000 \\
Biocontainment & $1(5)$ & $4(6)$ & 0.571 \\
\hline $\begin{array}{l}\text { Hospital admission assessed as necessary, } \\
\text { n. (\%) }\end{array}$ & $12(63)$ & $50(72)$ & \\
\hline
\end{tabular}

Abbreviations. IQR, inter-quartile range; n., number.

\section{Figures}


269 Children referred by

primary care pediatricians

Teleconsultation
with infectious disease specialist

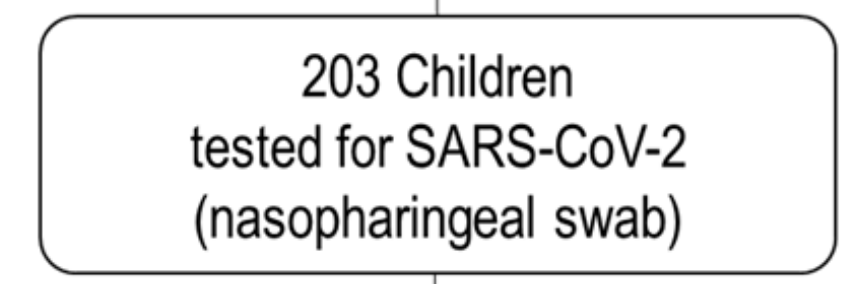

64 Negative for SARS-CoV-2

139 Children positive for SARS-CoV-2

\section{Children managed at home in telemedicine}

66 Children without exposure history or suggesting clinical features 